\title{
Antimicrobial use in acute care hospitals: national point prevalence survey on healthcare-associated infections and antimicrobial use, Switzerland, 2017
}

Walter Zingg ${ }^{1,2}$, Aliki Metsini' ${ }^{1}$, Céline Gardiol 3 , Carlo Balmelli4, Michael Behnke ${ }^{5}$, Nicolas Troillet ${ }^{6}$, Andreas Widmer $^{7}$, Didier

Pittet $^{1}$, on behalf of the Swissnoso Network ${ }^{8}$

1. Infection Control Programme and WHO Collaborating Centre on Patient Safety, University of Geneva Hospitals, Geneva, Switzerland

2. Imperial College London, London, United Kingdom

3. Swiss Federal Office of Public Health, Bern, Switzerland

4. Infection Control Programme, Cantonal Hospital Authority, Ticino, Switzerland

5. Institute of Hygiene and Environmental Medicine, Charité University Medicine Berlin, Berlin, Germany

6. Department of Infectious Diseases, Central Institute, Valais Hospital, Sion, Switzerland

7. Division of Infectious Diseases and Hospital Epidemiology, University Hospital Basel, Basel, Switzerland

8. Members of the network are acknowledged at the end of the article

Correspondence: Walter Zingg (walter.zingg@hcuge.ch)

Citation style for this article:

Zingg Walter, Metsini Aliki, Gardiol Céline, Balmelli Carlo, Behnke Michael, Troillet Nicolas, Widmer Andreas, Pittet Didier, on behalf of the Swissnoso Network. Antimicrobial use in acute care hospitals: national point prevalence survey on healthcare-associated infections and antimicrobial use, Switzerland, 2017. Euro Antimicrobial use in acute care hospitals: national point prevalence survey on healthcare-associate
Surveill. 2019;24(33):pii=1900015. https://doi.org/10.2807/1560-7917.ES.2019.24.33.1900015

Background: A point prevalence survey (PPS) on healthcare-associated infections ( $\mathrm{HAI}$ ) and antimicrobial use was conducted in Swiss acute care hospitals in 2017. Aim: Our objective was to assess antimicrobial use in Swiss acute care hospitals. Methods: All patients hospitalised in any acute care hospital in Switzerland were eligible. We used the most recent version of the PPS protocol of the European Centre for Disease Prevention and Control. Results: Data from 12,931 patients of 96 hospitals were collected. Of these, 4,265 (33\%; $95 \%$ confidence interval (Cl): $32.2-33.8)$ were on 5,354 antimicrobials for 4,487 indications. Most of the 2,808 therapeutic indications addressed 1,886 community-acquired infections (67.2\%; 95\% Cl: 65.4-68.9). Of the 1,176 surgical prophylaxes, 350 (29.8\%; $95 \% \mathrm{Cl}: 27.1-32.4)$ exceeded the duration of 1 day. Of the 1,090 antimicrobial regimens that were changed, 309 (28.3\%; $95 \%$ $\mathrm{Cl}$ : 25.7-31.0) were escalated and 337 (30.9\%; 95\% Cl: 28.2-33.7) were de-escalated. Amoxicillin/clavulanic acid was the most frequent antimicrobial (18.8\%; $95 \%$ $\mathrm{Cl}$ : 17.7-19.8), prescribed mainly for therapeutic indications $(76.0 \% ; 95 \% \mathrm{Cl}: 73.3-78.7)$. A total of 1,931 (37.4\%; $95 \% \mathrm{Cl}: 36.1-38.8)$ of the 5,158 antimicrobials for systemic use were broad-spectrum antibiotics, most frequently third- and fourth-generation cephalosporins (35.9\%; 95\% Cl: 33.8-38.1). Conclusions: Antimicrobial consumption was at European average, the use of broad-spectrum antibiotics in the lower third. Swiss acute care hospitals should invest in antimicrobial stewardship, particularly in reducing the use of broad-spectrum antibiotics.

\section{Introduction}

In the European Union (EU), one or more antimicrobials are given to at least a third of inpatients on any hospital day [1], which makes them one of the most frequently prescribed drug classes in acute care hospitals. Antimicrobial resistance (AMR) has become a global problem, and knowledge about the local and regional situation is important to guide therapeutic decisions [2]. Antimicrobials, particularly broad-spectrum antibiotics, drive the emergence of AMR, mostly through selection pressure [3,4], and favour infections caused by Clostridium difficile [5-7] or fungi [8]. Infections that are due to Gram-positive or Gram-negative multidrugresistant microorganisms (MDRO) prolong hospital stay and increase mortality [9-11]. Antimicrobial stewardship programmes, if resulting in measurable reduction and judicious use of antimicrobials, effectively reduce AMR [12].

For many years, point prevalence surveys (PPS) have been conducted for the surveillance of antimicrobial use, first in outpatient care [13], later in hospitals [14]. The European Surveillance of Antimicrobial Consumption Network (ESAC-Net) monitors the use of antimicrobials in the European Union (EU) and the European Economic Area (EEA), but does not provide clinical data to assess the appropriateness of antimicrobial prescriptions $[15,16]$. This gap was filled by combining prevalence surveys on healthcare-associated infections ( $\mathrm{HAl}$ ) and on antimicrobial use. In 2011 and 2012, the EU Member States, Iceland, Norway, and Croatia participated in such a combined PPS, the first 
PPS by the European Centre for Disease Prevention and Control (ECDC PPS) on HAl and antimicrobial use $[1,17]$. The HAl prevalence at that time was $6.0 \%$, and $35.0 \%$ of patients received one or more antimicrobials [1]. Five years later, the second ECDC PPS was conducted in the EU and EEA countries and in some EU candidate countries $[16,18,19]$. The HAI prevalence was $6.5 \%$, and $30.3 \%$ of patients received one or more antimicrobials. Switzerland was part neither of the first nor the second ECDC PPS.

In January 2013, the Swiss Federal Council passed its 'Health 2020' agenda to set priorities in healthcare management in Switzerland [20]. The Federal Office of Public Health (FOPH), together with various stakeholders from health delivery in Switzerland, defined two strategies for $\mathrm{HAl}$ prevention and AMR: the 'Strategy NOSO' and the 'Strategy StAR' [21,22]. Those national strategies aim at reducing HAls and containing the emergence and spread of AMR in the various healthcare settings in Switzerland. FOPH mandated Swissnoso, a non-profit association of leading experts in the field of infection prevention and control and infectious diseases in Switzerland ( www.swissnoso. ch ), to perform a national PPS (CH-PPS) on HAI and the use of antimicrobials, simultaneously with the second ECDC PPS and using the same protocol. The objective was to assess the situation of $\mathrm{HAl}$ and antimicrobial use in Swiss acute care hospitals [18].

\section{Methods}

\section{Setting and study population}

In 2016, a pilot survey was performed in three large acute care hospitals in Switzerland with the objective to test the ECDC PPS protocol [23]. The ECDC protocol version 5.3 was translated into French, German and Italian (www.swissnoso.ch) [18]. No methodological changes were made in the $\mathrm{CH}$-PPS protocol. In December 2016, all 187 acute care hospitals in Switzerland were invited to participate in the $\mathrm{CH}$-PPS, planned for the second trimester of 2017. Participation was voluntary, and hospitals were compensated for their efforts: CHF 200 (EUR 176) as a minimum fee plus CHF 5 (EUR 4.40) per additional included patient.

\section{Data collection}

The CH-PPS coordination centre was established for project management, education and training, data management, and data analysis. The centre organised seven interactive training workshops for hospital investigators and data collectors: four in the Germanspeaking region, two in the French-speaking region, and one in the Italian-speaking region of Switzerland. The courses used a structured methodology, encouraging a participative, problem-solving approach by discussing clinical cases and using the database interactively. The main data collection started on 1 April 2017 and ended on 30 June 2017, either using the specific $\mathrm{CH}$-PPS case report forms followed by data entry to the electronic $\mathrm{CH}$-PPS database or by direct data entry. The database featured plausibility algorithms and was provided by the Institute of Hygiene and Environmental Medicine at Charité Universitätsmedizin Berlin, Berlin, Germany [24]. During the data entry period, hospitals had the option to download their own data in different formats (HTML, CSV, pdf).

All patients hospitalised in acute care were eligible for inclusion if admitted to the ward before or at 08:00 and if not discharged during the survey day. Patients in the emergency room, in psychiatry and in outpatient care were excluded. Patients in rehabilitation and long-term care were included if such specialties were part of the acute care hospital. Patient information was collected using an individual patient form on demographic data, $\mathrm{HAl}$, medical device and antimicrobial use, and data on microorganisms with information on AMR. The detailed methodology is described elsewhere [23]. In addition, the hospitals provided data on antimicrobial policy, antimicrobial stewardship and additional activities in the context of antimicrobial use.

For antimicrobial use, the following data were collected: agent, route, dosage and indication as judged by the prescriber (treatment of community-, hospitalor long-term care-acquired infection, surgical or medical prophylaxis), diagnosis by anatomical site in case of treatment, documentation of the reason for antimicrobial prescription in the patient chart and change of the current antimicrobial regime. In case of changed regime, additional information on the last change was obtained: escalation, de-escalation, change from intravenous to oral, or any other type of change. The prevalence of antimicrobial use was reported as the percentage of patients receiving one or more antimicrobials on the survey day. The Anatomical Therapeutic Chemical (ATC) classification system was used for data analysis. Drugs were defined to the 5 th level of the ATC classification. The relative frequencies of individual antimicrobials representing the $75 \%$ most commonly used drugs (DU75\%) were calculated [25]. Defined daily doses (DDD) per 100 adult ( $\geq 18$ years) patient-days were calculated using the 2018 ATC/DDD classification issued by the World Health Organization Collaborating Centre for Drug Statistics and Methodology [26]. Outcomes were stratified by hospital size (small:<200 beds; medium size: 200-650 beds; large: $\mathbf{6 5 0}$ beds) and hospital type (primary care, secondary care, tertiary care, specialised care). We also calculated the proportion of broad-spectrum antibiotics among all antibiotics for systemic use (ATC J01). The following antimicrobial groups and agents were considered broad-spectrum antibiotics: piperacillin/tazobactam, third- and fourth-generation cephalosporins, monobactams, carbapenems, fluoroquinolones, glycopeptides, polymyxins, daptomycin and oxazolidinones [27].

\section{Data analysis}

Descriptive data are reported as medians with interquartile range (IQR) or means with $95 \%$ confidence intervals $(\mathrm{Cl})$ where appropriate. Statistical analysis 
TABLE 1

Antimicrobial use and hospital indicators on antimicrobial stewardship and surveillance, by hospital size and hospital category, national point prevalence survey on antimicrobial use, Switzerland, 2017 ( $\mathrm{n}=12,931$ patients)

\begin{tabular}{|c|c|c|c|c|c|c|c|c|c|c|c|}
\hline & \multirow{3}{*}{$\begin{array}{c}\text { Hospitals } \\
\text { n }\end{array}$} & \multirow{3}{*}{$\begin{array}{l}\text { Patients } \\
\left.\text { n (adults }{ }^{b}\right)\end{array}$} & \multicolumn{4}{|c|}{ Antimicrobial use } & \multicolumn{3}{|c|}{ Antimicrobial stewardship } & \multicolumn{2}{|c|}{ Surveillance ${ }^{a}$} \\
\hline & & & \multicolumn{3}{|c|}{$\begin{array}{l}\text { Patients } \\
\geq 1 \mathrm{AM}\end{array}$} & \multirow{2}{*}{$\begin{array}{c}\mathrm{DDD}^{\mathrm{b}} \\
\begin{array}{c}\mathrm{n} / 100 \\
\mathrm{PD}\end{array}\end{array}$} & \multirow{2}{*}{$\begin{array}{c}\text { AMS } \\
\text { consultant } \\
\text { FTE/250 } \\
\text { beds }^{d}\end{array}$} & \multirow{2}{*}{$\begin{array}{l}\text { Post- } \\
\text { prescription } \\
\text { reviewc } \\
\text { n/N }\end{array}$} & \multirow{2}{*}{$\begin{array}{c}\text { AM } \\
\text { guidelines } \\
\text { n/N }\end{array}$} & \multirow{2}{*}{$\begin{array}{l}\text { AM } \\
\text { use } \\
\text { n/N }\end{array}$} & \multirow{2}{*}{$\begin{array}{l}\text { AMR } \\
n / N\end{array}$} \\
\hline & & & $\mathrm{n}$ & $\%$ & $95 \% \mathrm{Cl}$ & & & & & & \\
\hline \multicolumn{12}{|l|}{ Hospital size } \\
\hline$<200$ beds & 63 & $3,516(3,155)$ & 1,175 & 33.4 & $31.9-35.0$ & 51.9 & 0.01 & $4 / 28$ & $40 / 63$ & $23 / 96$ & $36 / 96$ \\
\hline $200-650$ beds & 26 & $4,380(4,137)$ & 1,506 & 34.4 & $33.0-35.8$ & 57.7 & 0.03 & $5 / 16$ & $21 / 26$ & $13 / 96$ & $15 / 96$ \\
\hline$>650$ beds & 7 & $5,035(4,649)$ & 1,584 & 31.5 & $30.2-32.7$ & 66.1 & 0.01 & $0 / 2$ & $6 / 6$ & $7 / 96$ & $6 / 96$ \\
\hline \multicolumn{12}{|l|}{ Hospital type } \\
\hline Primary care & 38 & $2,694(2,510)$ & 865 & 32.1 & $30.3-33.9$ & 54.6 & 0.02 & $4 / 18$ & $28 / 38$ & $14 / 96$ & $22 / 96$ \\
\hline Secondary care & 40 & $4,325(4,085)$ & 1,465 & 33.9 & $32.5-35.3$ & 53.8 & 0.02 & $4 / 18$ & $30 / 40$ & $21 / 96$ & $24 / 96$ \\
\hline Tertiary care & 11 & $5,549(4,984)$ & 1,810 & 32.6 & $31.4-33.9$ & 67.6 & 0.01 & $0 / 4$ & $8 / 10$ & $8 / 96$ & $9 / 96$ \\
\hline Specialised care & 7 & $363(362)$ & 125 & 34.4 & $29.5-39.3$ & 45.0 & 0.00 & $1 / 6$ & $1 / 7$ & $0 / 96$ & $2 / 96$ \\
\hline Total & 96 & $12,931(11,941)$ & 4,265 & 33.0 & 32.2-33.8 & 59.4 & 0.02 & $9 / 46$ & $67 / 95$ & $43 / 96$ & $57 / 96$ \\
\hline
\end{tabular}

AM: antimicrobial; AMR: antimicrobial resistance; AMS: antimicrobial stewardship; CI: confidence interval; DDD: defined daily doses; FTE: fulltime equivalent; PD: patient-days.

a Participation in a surveillance network.

b Patients $\geq 18$ years.

c Formal post-prescription review after 72-96 h in any setting (or hospital-wide); data provided by 46 hospitals only.

${ }^{d}$ Only six hospitals had a formal AMS consultant.

of patient characteristics, frequencies of indications and therapeutic diagnoses, and frequencies of broadspectrum antibiotic use relative to hospital size and types was done by using the non-parametric KruskalWallis test. A rapidly or ultimately fatal McCabe score (expected fatal outcome within 1 or 5 years, respectively) [28], age groups (0-17, 18-40, 41-60, $61-80,>80$ years), hospitalisation in an intensive care unit (ICU) on the survey day, exposure to a medical device (peripheral or central venous catheter, urinary catheter, endotracheal tube) on the survey day, having undergone National Healthcare Safety Network (NHSN) surgery [29] since hospital admission and private-forprofit ownership of the hospital were tested in a univariable logistic regression analysis as risk factors for both receiving one or more antimicrobials and receiving systemic broad-spectrum antibiotics. Variables with a significance level of $p \leq 0.2$ were tested in a multivariable model. Observations were clustered at hospital level; a two-sided $p$ value of 0.05 was considered significant. Data analysis was performed using STATA version 13 (STATA Corporation, College Station, United States).

\section{Ethical statement}

No institutional review board approval was deemed necessary, similar to the ECDC PPS, and given the quality improvement character of the survey. Only anonymous patient and ward data were collected and analysed.

\section{Results}

Ninety-six hospitals with 12,931 patients participated in the survey: $63(65.6 \%)$ small hospitals with 3,516 (27.2\%) patients, 26 (27.1\%) medium-size hospitals with 4,380 (33.9\%) patients, and 7 (7.3\%) large hospitals with 5,035 (38.9\%) patients [30]. Median age was 68 years (IQR: $48-80$ ). Patient characteristics of the participants of the national PPS are published elsewhere [30].

Table 1 summarises antimicrobial use and hospital indicators on antimicrobial stewardship and surveillance, stratified by hospital size and hospital category. A total of 4,265 patients received one or more antimicrobials on the survey day, resulting in a prevalence of $33.0 \%(95 \% \mathrm{Cl}: 32.2-33.8)$. The prevalence of patients on one or more antimicrobials in small, medium size and large hospitals was $33.4 \%$ ( $n=1,175 ; 95 \% \mathrm{Cl}: 31.9-35.0), 34.4 \% \quad(n=1,506 ; 95 \%$ $\mathrm{Cl}: 33.0-35.8)$ and $31.5 \%(n=1,584 ; 95 \% \mathrm{Cl}: 30.2-$ $32.7)$, respectively $(p=0.009)$. Of the 358 paediatric patients, 69 (19.3\%; 95\% Cl: 15.3-23.7) received one or more antimicrobials on the survey day, while this proportion was $33.4 \%$ (95\% Cl: $32.6-34.2)$ in adults. Overall DDD were 59.4 per 100 patients $(7,089$ DDD in 11,941 adults). Re-evaluation of antimicrobial treatment after $48-72 \mathrm{~h}$ was performed by nine of the 46 hospitals reporting on this indicator. Only six hospitals (two per hospital size category) had formal positions for antimicrobial stewardship. Guidelines on antimicrobial use were available in $70.5 \%$ of 95 hospitals reporting 
Indications for antimicrobial use and diagnoses for therapeutic treatment, by hospital size, national point prevalence survey on antimicrobial use, Switzerland, $2017(\mathrm{n}=4,487)$

\begin{tabular}{|c|c|c|c|c|c|c|c|c|c|c|c|c|c|}
\hline & \multirow{2}{*}{\multicolumn{3}{|c|}{ All hospitals }} & \multicolumn{9}{|c|}{ Hospital size } & \multirow{3}{*}{ p value } \\
\hline & & & & \multicolumn{3}{|c|}{$<200$ beds } & \multicolumn{3}{|c|}{$200-650$ beds } & \multicolumn{3}{|c|}{$>650$ beds } & \\
\hline & $\mathrm{n}$ & $\%$ & $95 \% \mathrm{Cl}$ & $n$ & $\%$ & $95 \% \mathrm{Cl}$ & $\mathrm{n}$ & $\%$ & $95 \% \mathrm{Cl}$ & $\mathrm{n}$ & $\%$ & $95 \% \mathrm{Cl}$ & \\
\hline Indications & \multicolumn{3}{|c|}{$n=4,487$} & \multicolumn{3}{|c|}{$\mathrm{n}=1,216$} & \multicolumn{3}{|c|}{$n=1,545$} & \multicolumn{3}{|c|}{$\mathrm{n}=1,726$} & \\
\hline $\begin{array}{l}\text { Community-acquired } \\
\text { infection }\end{array}$ & 1,886 & 42.0 & $40.6-43 \cdot 5$ & 513 & 42.2 & $39 \cdot 4-45 \cdot 0$ & 726 & 47.0 & $44 \cdot 5-49 \cdot 5$ & 647 & $37 \cdot 5$ & $35.2-39.8$ & $<0.001$ \\
\hline $\begin{array}{l}\text { Healthcare-associated } \\
\text { infection }^{\mathrm{a}}\end{array}$ & 852 & 19.0 & $17.8-20.1$ & 173 & 14.2 & $12.2-16.2$ & 245 & 15.9 & $14 \cdot 0-17 \cdot 7$ & 434 & 25.1 & $23.1-27.2$ & $<0.001$ \\
\hline $\begin{array}{l}\text { LTCF-acquired } \\
\text { infection }\end{array}$ & 70 & 1.6 & $1.2-1.9$ & 25 & 2.1 & $1.3-2.9$ & 24 & 1.6 & $0.9-2.2$ & 21 & 1.2 & $0.7-1.7$ & 0.280 \\
\hline Medical prophylaxis & 357 & 8.0 & $7.2-8.7$ & 44 & 3.6 & $2.6-4 \cdot 7$ & 74 & 4.8 & $3.7-5.9$ & 239 & 13.8 & $12.2-15.5$ & $<0.001$ \\
\hline Surgical prophylaxis & 1,176 & 26.2 & $24.9-27.5$ & 437 & $35 \cdot 9$ & $33.2-38.6$ & 424 & 27.4 & $25.2-29.7$ & 315 & 18.3 & $16.4-20.1$ & $<0.001$ \\
\hline $\begin{array}{l}\text { Other or unknown } \\
\text { indication }\end{array}$ & 146 & $3 \cdot 3$ & $2.7-3.8$ & 24 & 2.0 & $1.2-2.8$ & 52 & 3.4 & $2.5-4.3$ & 70 & 4.1 & $3.1-5.0$ & 0.007 \\
\hline Diagnoses $^{\mathrm{b}}$ & \multicolumn{3}{|c|}{$\mathrm{n}=2,808$} & \multicolumn{3}{|c|}{$\mathrm{n}=711$} & \multicolumn{3}{|c|}{$n=995$} & \multicolumn{3}{|c|}{$n=1,102$} & \\
\hline $\begin{array}{l}\text { Bloodstream } \\
\text { infection/sepsis }\end{array}$ & 286 & 10.2 & $9.1-11.4$ & 56 & $7 \cdot 9$ & $6.0-10.1$ & 109 & 11.0 & $9.1-13.1$ & 121 & 11.0 & $9.2-13.0$ & 0.013 \\
\hline $\begin{array}{l}\text { Lower respiratory } \\
\text { tract infection }\end{array}$ & 678 & 24.1 & $22.6-25.8$ & 173 & $24 \cdot 3$ & $21.2-27.7$ & 232 & $23 \cdot 3$ & $20.7-26.1$ & 273 & 24.8 & $22.2-27.4$ & 0.491 \\
\hline Urinary tract infection & 448 & 16.0 & $14 \cdot 6-17.4$ & 151 & 21.3 & $18 \cdot 3-24 \cdot 4$ & 164 & 16.5 & $14 \cdot 2-18.9$ & 133 & 12.1 & $10.2-14.1$ & $<0.001$ \\
\hline Soft tissue infection & 276 & 9.8 & $8.8-11.0$ & 78 & 11.0 & $8.8-13.5$ & 118 & 11.9 & $9.9-14.0$ & 80 & $7 \cdot 3$ & $5.8-9.0$ & 0.002 \\
\hline $\begin{array}{l}\text { Bone and joint } \\
\text { infection }\end{array}$ & 140 & 5.0 & $4 \cdot 2-5 \cdot 9$ & 48 & 6.8 & $5.0-8.9$ & 33 & $3 \cdot 3$ & $2.3-4.6$ & 59 & $5 \cdot 4$ & $4.1-6.9$ & 0.017 \\
\hline Surgical site infection & 250 & 8.9 & $7.9-10.0$ & 52 & $7 \cdot 3$ & $5 \cdot 5-9 \cdot 5$ & 90 & 9.0 & $7.3-11.0$ & 108 & 9.8 & $8.1-11.7$ & 0.061 \\
\hline $\begin{array}{l}\text { Intra-abdominal } \\
\text { infection }\end{array}$ & 266 & $9 \cdot 5$ & $8.4-10.6$ & 59 & 8.3 & $6.4-10.6$ & 101 & 10.2 & $8.3-12.2$ & 106 & 9.6 & $7 \cdot 9-11.5$ & 0.158 \\
\hline $\begin{array}{l}\text { Gastrointestinal } \\
\text { infection }\end{array}$ & 116 & 4.1 & $3 \cdot 4-4 \cdot 9$ & 28 & $3 \cdot 9$ & $2.6-5.6$ & 38 & 3.8 & $2.7-5.2$ & 50 & $4 \cdot 5$ & $3 \cdot 4-5 \cdot 9$ & 0.565 \\
\hline Vascular infection & 71 & 2.5 & $2.0-3.2$ & 10 & 1.4 & $0.7-2.6$ & 24 & 2.4 & $1.6-3.6$ & 37 & 3.4 & $2.4-4.6$ & 0.829 \\
\hline Other infection & 277 & 9.9 & $8.8-11.0$ & 56 & $7 \cdot 9$ & $6.0-10.1$ & 86 & 8.6 & $7.010 .6-$ & 135 & 12.3 & $10.4-14 \cdot 3$ & 0.001 \\
\hline
\end{tabular}

$\mathrm{Cl}$ : confidence interval; LTCF: long-term care facility.

a Healthcare-associated as documented in the patient chart.

${ }^{b}$ Diagnoses: applicable to community-acquired infections, healthcare-associated infections, and LTCF-acquired infections (2,808 in total).

on this indicator, with higher proportions in large and tertiary care hospitals. Surveillance of AMR and antimicrobial use was reported by $57(59.4 \%)$ and $43(44.8 \%)$ hospitals, respectively; again more often in large and tertiary care hospitals.

The 4,265 patients on antimicrobials received 5,354 antimicrobial agents for 4,487 indications. Table 2 summarises indications for antimicrobial use and diagnoses for therapeutic indications. Numbers and proportions of indications for therapeutic use, prophylaxis and other or unknown indications were 2,808 (62.6\%; 95\% Cl: 61.2-64.0), 1,533 (34.2\%; 95\% Cl: $32.8-35.6)$ and 146 (3.3\%; 95\% Cl: 2.7-3.8), respectively. Most of the 2,808 therapeutic indications were for community-acquired infections ( $n=1,886$; 67.2\%; 95\% Cl: 65.4-68.9) (Supplementary Table 1) and predominantly for treating lower respiratory tract $(n=678 ; 24.1 \% ; 95 \% \quad C l: 22.6-25.8)$, urinary tract $(n=448 ; 16.0 \% ; 95 \% \mathrm{Cl}: 14.6-17.4)$ and bloodstream infections ( $\mathrm{n}=286 ; 10.2 \% ; 95 \% \mathrm{Cl}$ : 9.1-11.4) (Table 2). Most of the 1,533 prophylactic indications were for surgical procedures ( $\mathrm{n}=1,176 ; 76.7 \% ; 95 \% \mathrm{Cl}: 74.6-78.8)$, of which 677 (57.6\%; 95\% Cl: 54.7-60.4) were administered as single dose, 149 (12.7\%; 95\% Cl: 10.8-14.6) during 1 day and 350 (29.8\%; 95\% Cl: 27.1-32.4) for more than 1 day (Supplementary Table 1 ). While the proportion of prolonged surgical prophylaxis in small hospitals (87/437; 19.9\%; 95\% Cl: 16.1-23.7) and medium-size hospitals (104/424; $24.5 \% ; 95 \% \mathrm{Cl}$ : 20.4-28.6) was small, this proportion was statistically significantly higher in large hospitals (159/315; 50.5\%; $95 \% \mathrm{Cl}: 44.9-56.0)$. A total of 1,542 (28.8\%; $27.6-30.0)$ of the 5,354 antimicrobials were administered by the oral route, with the highest proportion in rehabilitation (59/84; 70.2\%; 95\% Cl: 60.3-80.2), followed by geriatrics (70/133; 52.6\%; 95\% Cl: 44.0-61.2) and internal medicine (814/2,066; 39.4\%; 95\% Cl: 37.3-41.5). 
TABLE 3

Change of antimicrobial treatment, national point prevalence survey on antimicrobial use, Switzerland, 2017 $(\mathrm{n}=4,487)$

\begin{tabular}{|c|c|c|c|c|c|c|c|c|c|c|c|c|c|}
\hline & \multirow{2}{*}{$\begin{array}{c}\text { Indications } \\
\text { total }\end{array}$} & \multicolumn{3}{|c|}{ Reason in notes } & \multicolumn{3}{|c|}{ Any change } & \multicolumn{3}{|c|}{ Escalation } & \multicolumn{3}{|c|}{ De-escalation } \\
\hline & & $n$ & $\begin{array}{l}\% \text { of } \\
\text { total }\end{array}$ & $95 \% \mathrm{Cl}$ & $\mathrm{n}$ & $\begin{array}{l}\% \text { of } \\
\text { total }\end{array}$ & $95 \% \mathrm{Cl}$ & $\mathrm{n}$ & $\begin{array}{l}\% \text { of } \\
\text { change }\end{array}$ & $95 \% \mathrm{Cl}$ & $\mathrm{n}$ & $\begin{array}{l}\% \text { of } \\
\text { change }\end{array}$ & $95 \% \mathrm{Cl}$ \\
\hline \multicolumn{14}{|l|}{ Hospital size } \\
\hline <200 beds & 1,216 & 1,040 & 85.5 & $83.5-87.5$ & 249 & 20.5 & $18.2-22.7$ & 77 & 30.9 & $25.1-36.7$ & 63 & $25 \cdot 3$ & $19.9-30.7$ \\
\hline $200-650$ beds & 1,545 & 1,355 & 87.7 & $86.1-89.3$ & 381 & 24.7 & $22.5-26.8$ & 87 & 22.8 & $18.6-27.1$ & 126 & 33.1 & $28.3-37.8$ \\
\hline$>650$ beds & 1,726 & 1,508 & 87.4 & $85.8-88.9$ & 460 & 26.7 & $24.6-28.7$ & 142 & 30.9 & $26.6-35.1$ & 155 & 33.7 & $29.4-38.0$ \\
\hline \multicolumn{14}{|l|}{ Hospital type } \\
\hline Primary care & 886 & 759 & 85.7 & $83.4-88.0$ & 188 & 21.2 & $18.5-23.9$ & 58 & 30.9 & $24.2-37.5$ & 46 & 24.5 & $18.3-30.7$ \\
\hline Secondary care & 1,510 & 1,323 & 87.6 & $86.0-89.3$ & 369 & 24.4 & $22.3-26.6$ & 86 & 23.3 & $19.0-27.6$ & 127 & 34.4 & $29.5-39.3$ \\
\hline Tertiary care & 1,956 & 1,693 & 86.6 & $85.0-88.1$ & 511 & 26.1 & $24.2-28.1$ & 159 & 31.1 & $27.1-35.1$ & 169 & 33.1 & $29.0-37.2$ \\
\hline Specialised care & 135 & 128 & 94.8 & $91.0-98.6$ & 22 & 16.3 & $10.0-22.6$ & 3 & 13.6 & $0.0-29.2$ & 2 & 9.1 & $0.0-22.1$ \\
\hline \multicolumn{14}{|c|}{ Indication/diagnosis } \\
\hline Therapy & 2,808 & 2,638 & 94.0 & 93.1-94.8 & 1,023 & 36.4 & $34.7-38.2$ & 306 & 29.9 & $27.1-32.7$ & 344 & 33.6 & $30.7-36.5$ \\
\hline $\begin{array}{l}\text { Community } \\
\text { infection }\end{array}$ & 1,886 & 1,781 & 94.4 & $93.4-95.5$ & 652 & 34.6 & $32.4-36.7$ & 163 & 25.0 & $21.7-28.3$ & 235 & 36.0 & $32.3-37.7$ \\
\hline $\begin{array}{l}\text { Healthcare- } \\
\text { associated } \\
\text { infection }\end{array}$ & 852 & 795 & 93.3 & $91.6-95.0$ & 342 & 40.1 & $36.8-43.4$ & 136 & 39.8 & $\begin{array}{c}34.6- \\
45.0\end{array}$ & 101 & 29.5 & $24 \cdot 7-34.4$ \\
\hline $\begin{array}{l}\text { Bloodstream } \\
\text { infection/sepsis }\end{array}$ & 286 & 268 & 93.7 & $90.9-96.5$ & 126 & 44.1 & $38.3-49.8$ & 31 & 24.6 & $17.0-32.2$ & 54 & 42.9 & $34.1-51.6$ \\
\hline $\begin{array}{l}\text { Lower respiratory } \\
\text { tract infection }\end{array}$ & 678 & 645 & 95.1 & $93.5-96.8$ & 263 & 38.8 & $35.1-42.5$ & 80 & 30.4 & $\begin{array}{c}24.8- \\
36.0 \\
\end{array}$ & 73 & 27.8 & $22.3-33.2$ \\
\hline $\begin{array}{l}\text { Urinary tract } \\
\text { infection }\end{array}$ & 448 & 423 & $94 \cdot 4$ & $92.3-96.6$ & 129 & 28.8 & $24.6-33.0$ & 38 & 29.5 & $21.5-37.4$ & 49 & 38.0 & $29.5-46.5$ \\
\hline $\begin{array}{l}\text { Intra-abdominal } \\
\text { infection }\end{array}$ & 266 & 244 & 91.7 & $88.4-95.1$ & 108 & 40.6 & $34.7-46.5$ & 40 & 37.0 & $27.8-46.3$ & 35 & 32.4 & $23.4-41.4$ \\
\hline $\begin{array}{l}\text { Surgical site } \\
\text { infection }\end{array}$ & 250 & 233 & 93.2 & $90.1-96.3$ & 129 & 51.6 & $45.4-57.8$ & 43 & 33.3 & $25.1-41.6$ & 35 & 27.1 & $19.4-34.9$ \\
\hline \multicolumn{14}{|l|}{ Patient specialty } \\
\hline Intensive care & 204 & 179 & 87.8 & $83.2-92.3$ & 64 & 31.4 & $25.0-37.8$ & 25 & 39.1 & $26.8-51.3$ & 24 & 37.5 & $25.3-49.7$ \\
\hline Surgery & 2,133 & 1,789 & 83.9 & $82.3-85.4$ & 418 & 19.6 & $17.9-21.3$ & 118 & 28.2 & $23.9-32.6$ & 121 & 29.0 & $24.6-33.3$ \\
\hline Internal medicine & 1,680 & 1,542 & 91.8 & $90.5-93.1$ & 528 & 31.4 & $29.2-33.7$ & 148 & 28.0 & $24.2-31.9$ & 175 & 33.1 & $29.1-37.2$ \\
\hline Paediatrics & 69 & 55 & 79.7 & $70.0-89.4$ & 9 & 13.0 & $4.9-21.2$ & 2 & 22.2 & $0.0-56.1$ & 2 & 22.2 & $0.0-56.1$ \\
\hline Geriatrics & 115 & 11 & 9.6 & $4.9-16.5$ & 31 & 27.0 & $18.7-35.2$ & 6 & 19.4 & $4.6-34.1$ & 11 & 35.5 & $17.6-53.3$ \\
\hline Rehabilitation & 71 & 59 & 83.1 & $74.2-92.0$ & 20 & 28.2 & $17.4-38.9$ & 2 & 10.0 & $0.0-24.4$ & 6 & 30.0 & $8.0-52.0$ \\
\hline Total & 4,487 & 3,903 & 87.0 & 86.0-88.9 & 1,090 & $24 \cdot 3$ & $23.0-25.5$ & 309 & 28.3 & $25.7-31.0$ & 337 & 30.9 & 28.2-33.7 \\
\hline
\end{tabular}

A quarter of antimicrobial regimens were changed (1,090/4,487; $24.3 \% ; 95 \% \mathrm{Cl}: 23.0-25.5)$. Most often, change occurred in surgical site $(129 / 250 ; 51.6 \% ; 95 \%$ $\mathrm{Cl}$ : 45.4-57.8), bloodstream (126/286; 44.1\%; $95 \% \mathrm{Cl}$ : 38.3-49.8), intra-abdominal (108/266; 40.6\%; $95 \%$ $\mathrm{Cl}: 34.7-46.5)$ and healthcare-associated infections (342/852; 40.1\%; 95\% Cl: 36.8-43.4) (Table 3). A total of 309 (28.3\%; $95 \% \mathrm{Cl}: 25.7-31.0)$ and $337(30.9 \%$; $95 \% \mathrm{Cl}: 28.2-33.7)$ of the 1,090 changed antimicrobial regimens were escalated and de-escalated, respectively. Escalation was most often reported in intensive care $(25 / 64 ; 39.1 \% ; 95 \% \mathrm{Cl}: 26.8-51.3)$, healthcareassociated (136/342; 39.8\%; $95 \% \mathrm{Cl}: 34.6-45.0)$ and intra-abdominal infections (40/108; $37.0 \% ; 95 \% \mathrm{Cl}$ : 27.8-46.3) (Table 3). De-escalation was reported most often for bloodstream (54/126; 42.9; $95 \% \mathrm{Cl}$ : 34.151.6) and urinary tract infections (49/129; 38.0\%; $95 \%$
Cl: 29.5-46.5) (Table 3). Of the 3,479 antimicrobials prescribed for the 2,808 therapeutic indications, 255 ( $7.3 \% ; 95 \% \mathrm{Cl}: 6.5-8.2)$ were switched from intravenous to oral and $40(1.2 \% ; 95 \% \mathrm{Cl}: 0.8-1.5)$ were changed because of side effects. The majority of 3,903 $(87.0 \%$; $95 \% \mathrm{Cl}: 86.0-88.0$ ) of the 4,487 antimicrobial indications were documented in the patient charts, without differences across hospital size; however, specialised hospitals documented significantly more indications (128/135; 94.8\%; 95\% Cl: 91.0-98.6) compared with other hospital types (Table 3).

Figure 1 summarises the DU $75 \%$, stratified according to therapeutic use, surgical prophylaxis and medical prophylaxis. Amoxicillin/clavulanic acid was the most common antimicrobial drug $(1,006 / 5,354 ; 18.8 \% ; 95 \%$ $\mathrm{Cl}$ 17.7-19.8), used mainly for therapeutic indications 


\section{FIGURE 1}

Antimicrobials accounting for 75\% of antimicrobial use (DU 75\%), national point prevalence survey on antimicrobial use, Switzerland, $2017(\mathrm{n}=5,354)$

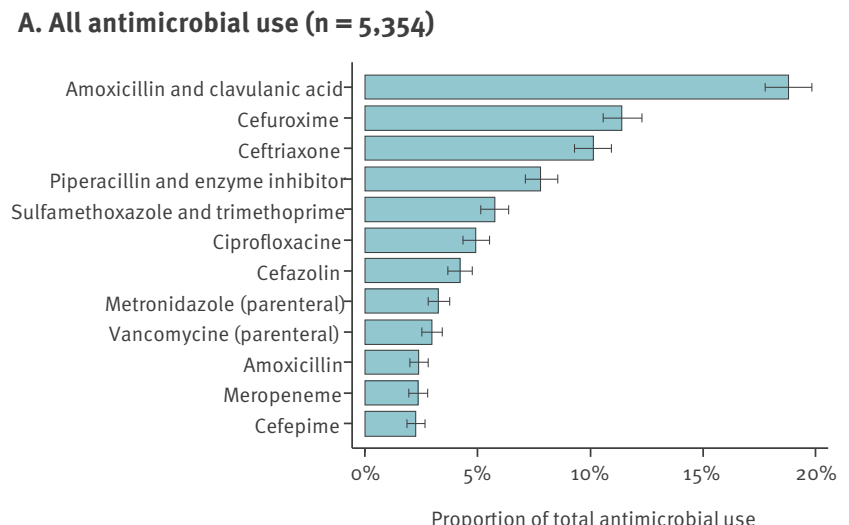

C. Antimicrobials for surgical prophylaxis $(n=1,176)$

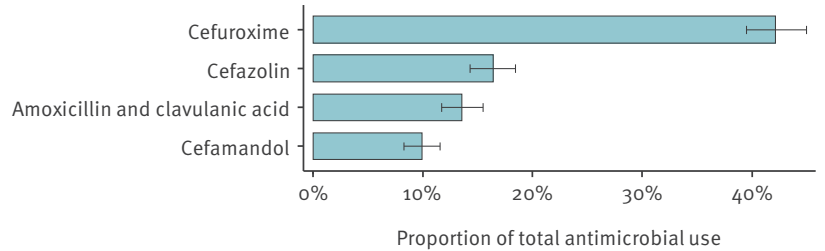

B. Antimicrobials for therapeutic use $(n=2,808)$

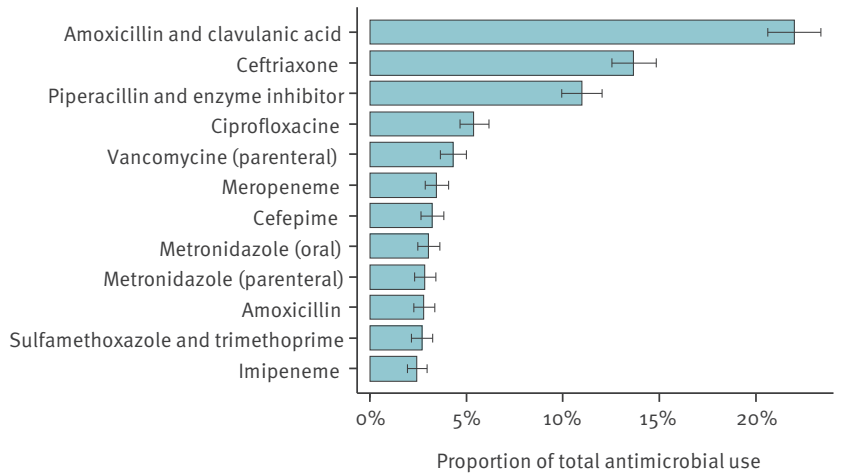

D. Antimicrobials for medical prophylaxis $(n=357)$

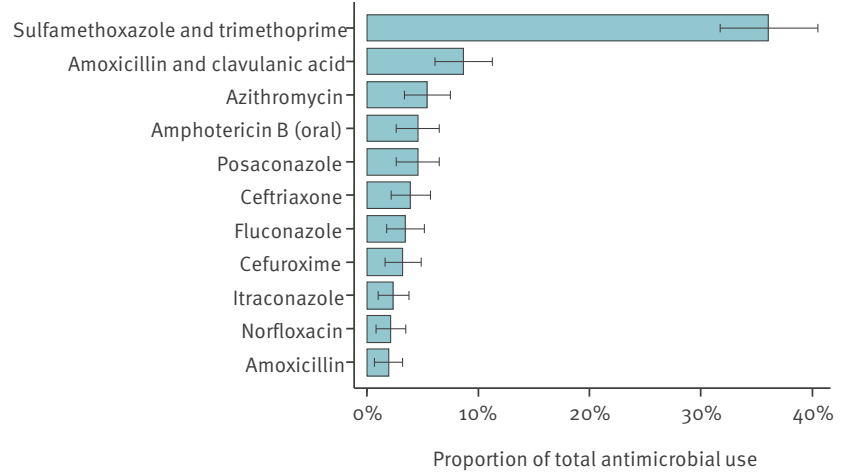

Bars and whiskers indicate antimicrobial use with $95 \%$ confidence intervals.

(765/1,006; 76.0\%; 95\% Cl: 73.3-78.7) such as community-acquired lower respiratory tract infection (235/765; $30.7 \%$; $95 \% \mathrm{Cl}: 27.5-34.1)$, followed by cefuroxime (612/5,354; $11.4 \% ; 95 \% \mathrm{Cl}: 10.6-12.3)$, used mainly for surgical prophylaxis (528/612; 86.3\%; 95\% Cl: $83.3-$ 88.9), followed by ceftriaxone $(542 / 5,354 ; 10.1 \%$; $95 \%$ $\mathrm{Cl}$ : 9.3-10.9), used mainly for therapeutic indications (476/542; 87.8\%; 95\% Cl: 84.8-90.5) such as community-acquired urinary $(145 / 476 ; 30.5 \% ; 95 \% \mathrm{Cl}: 26.4-$ 34.8) and lower respiratory tract infection (105/476; 22.1\%; 95\% Cl: 18.4-26.1) (Supplementary Table 2). Combination of two or more than two antimicrobial agents was reported for, respectively, 638 (14.2\%; 95\% $\mathrm{Cl}: 13.2-15.2)$ and $100(2.2 \% ; 95 \% \mathrm{Cl}: 1.8-2.7)$ of the 4,487 indications. The details of combined use of antimicrobial agents are summarised in Supplementary Table 3.

A total of 5,158 of the 5,354 antimicrobials were for systemic use (96.3\%; 95\% Cl: 95.8-96.8). Figure 2 summarises the proportions of broad-spectrum antibiotics among all antimicrobials for systemic use, stratified by hospital size and category as well as therapeutic use for community- and healthcareacquired infections. A total of 1,931 (37.4\%; $95 \% \mathrm{Cl}$ :
36.1-38.8) antimicrobials were broad-spectrum antibiotics, most commonly third- and fourth-generation cephalosporins (694/1,931; 35.9\%; 95\% Cl: 33.8-38.1), followed by piperacillin/tazobactam (419/1,931; $21.7 \%$; 95\% Cl: 19.9-23.6) and quinolones (347/1,931; 18.0\%; 95\% Cl: 16.3-19.8). Significantly more broad-spectrum antibiotics were used to treat HAl $(606 / 1,025 ; 59.1 \%$; $95 \% \mathrm{Cl}: 56.1-62.1)$ than to treat community-acquired infections (1,043/2,337; 44.6\%; 95\% Cl: 44.6-48.7) (Figure 2).

Table 4 summarizes uni- and multivariable analyses to predict antmimicrobial use. Male sex, exposure to one or more medical devices, undergoing NHSN-surgery and hospitalisation in intensive care were independently associated with receiving one or more antimicrobials (Table 4). Older age, exposure to one or more medical devices, rapidly or ultimately fatal McCabe scores and admission to intensive care were independently associated with receiving broad-spectrum antibiotics (Table 4). 
Proportion of broad-spectrum antibiotics among all antimicrobials for systemic use, national point prevalence survey on antimicrobial use, Switzerland, $2017(\mathrm{n}=5,158)$

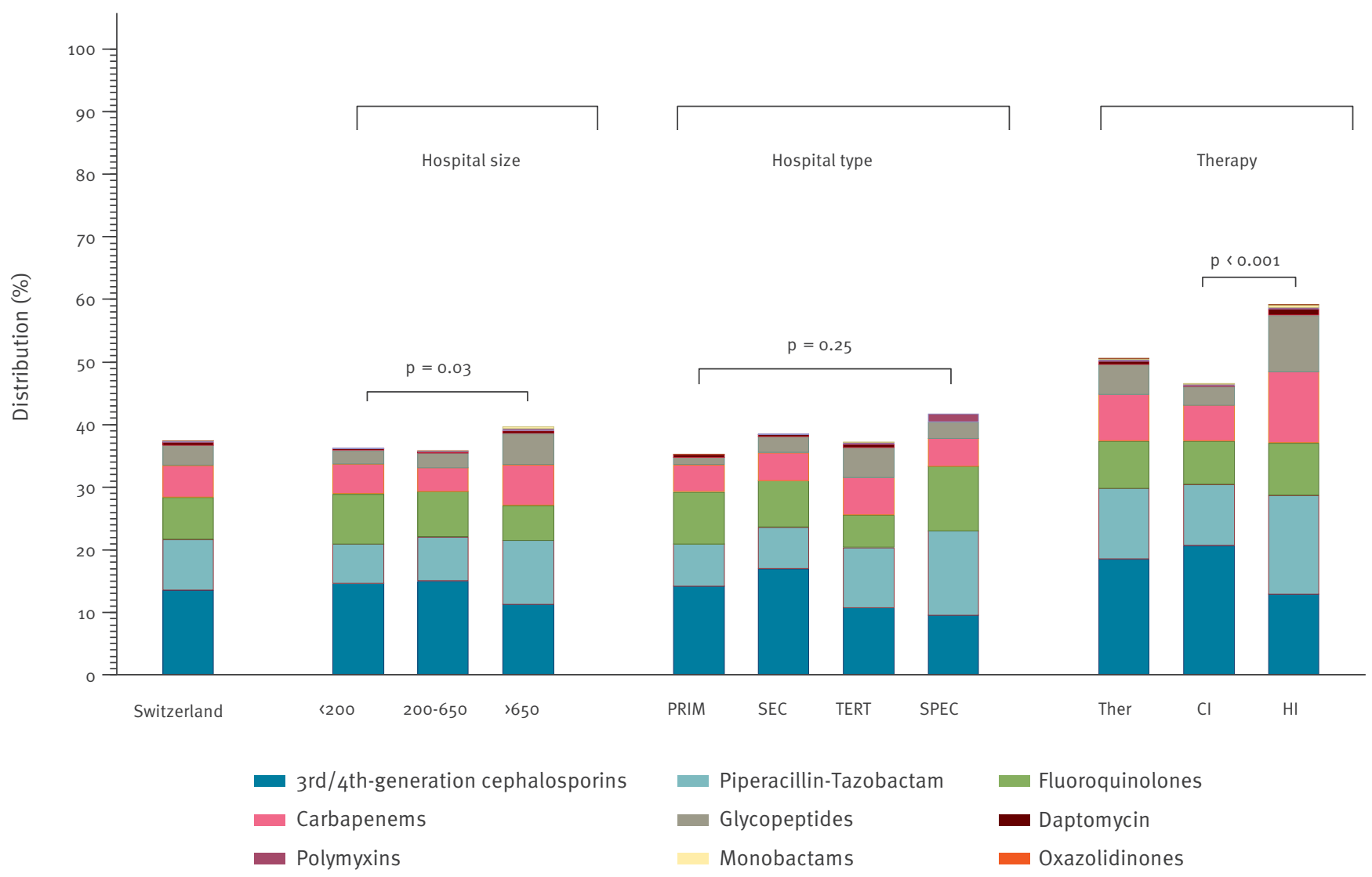

Cl: community infection; HI: healthcare-associated infection; SEC: secondary care; SPEC: specialty care; TERT: tertiary care; Ther: therapeutic use.

\section{Benchmarking to the second ECDC point} prevalence survey

Switzerland used the ECDC protocol and conducted the CH-PPS at the same time as the second ECDC PPS. Thus, data could be benchmarked to the EU and EEA countries. Figure 3 shows the position of Switzerland compared with the countries participating in the second ECDC PPS [16]. Antimicrobial use in Swiss acute care hospitals was at EU average (30.3\%).

\section{Discussion}

This was the first national PPS on antimicrobial use in Swiss acute care hospitals. The data are representative for Switzerland, considering that almost $80 \%$ of the acute care hospitals with 100 beds or more contributed data to the survey. One in three patients in acute care hospitals received one or more antimicrobials, of which nearly $40 \%$ were broad-spectrum antibiotics. The most important indication for antimicrobial use was the treatment of community-acquired infections, mainly lower respiratory and urinary tract infections. One in five patients received antimicrobials for the treatment of an HAl, mostly broad-spectrum antibiotics. Surgical prophylaxis was the second most common indication for the use of antimicrobials, of which a third was prescribed for more than 1 day.

Antimicrobial consumption is also monitored by anresis, a comprehensive and representative surveillance system for antibiotic consumption and resistance in Switzerland ( www.anresis.ch ) collecting data from hospital pharmacies. The quantities of antimicrobials in grams are converted to DDD and expressed as DDD per 100 bed-days [31]. In 2017, 62 DDD per 100 bed-days were reported, which is very close to the 59 DDDs per 100 patient-days measured in our survey [32]. Anresis covers surveillance of AMR for most of the Swiss acute care hospitals, while surveillance of antimicrobial consumption is done by a voluntary sentinel network of 70 hospital pharmacies. Anresis does not provide clinical data. Furthermore, although the system observes both consumption and resistance, no patient-based link is provided to put antimicrobial use into clinical perspective. Compared with Europe, Switzerland had low formal staffing for antimicrobial stewardship (0.02/250 beds vs $0.37 / 250$ beds), and a low proportion of hospitals with established formal post-prescription review (20\% vs $52 \%)$. 
TABLE 4

Predictive variables for the use of one or more antimicrobials and for broad-spectrum antibiotics, national point prevalence survey on antimicrobial use, Switzerland, $2017(\mathrm{n}=12,931)$

\begin{tabular}{|c|c|c|c|c|c|c|}
\hline \multirow{2}{*}{ Variable } & \multicolumn{3}{|c|}{ Univariable analysis } & \multicolumn{3}{|c|}{ Multivariable analysis } \\
\hline & OR & $95 \% \mathrm{Cl}$ & $\mathrm{p}$ value & OR & $95 \% \mathrm{Cl}$ & $\mathrm{p}$ value \\
\hline \multicolumn{7}{|l|}{ One or more antimicrobials } \\
\hline $\mathrm{Age}^{\mathrm{a}}$ & 1.07 & $1.00-1.13$ & 0.037 & & & \\
\hline Male sex & 1.53 & $1.40-1.66$ & $<0.001$ & 1.37 & $1.25-1.49$ & $<0.001$ \\
\hline Fatal McCabe score ${ }^{b}$ & 1.24 & $1.07-1.45$ & $<0.001$ & & & \\
\hline Medical device $^{c}$ & 6.75 & $6.00-7.65$ & $<0.001$ & 6.21 & $5.49-7.02$ & $<0.001$ \\
\hline NHSN surgery since admission & 1.78 & $1.56-2.03$ & $<0.001$ & 1.63 & $1.44-1.84$ & $<0.001$ \\
\hline Hospitalisation in intensive care & 3.48 & $2.74-4.42$ & $<0.001$ & 1.77 & $1.44-2.18$ & $<0.001$ \\
\hline Large hospital (>650 beds) & 0.89 & $0.65-1.23$ & 0.487 & & & \\
\hline Tertiary care hospital & $0.9)$ & $0.72-1.31$ & 0.851 & & & \\
\hline Private-for-profit ownership & 0.89 & $0.66-1.20$ & 0.437 & & & \\
\hline \multicolumn{7}{|l|}{ Broad-spectrum antibiotics } \\
\hline Age $^{\mathrm{a}}$ & 1.22 & $1.13-1.33$ & $<0.001$ & 1.17 & $1.09-1.26$ & $<0.001$ \\
\hline Male sex & 1.58 & $1.43-1.74$ & $<0.001$ & 1.33 & $1.21-1.46$ & $<0.001$ \\
\hline Fatal McCabe score ${ }^{\mathrm{b}}$ & 1.69 & $1.43-1.99$ & $<0.001$ & 1.36 & $1.16-1.60$ & $<0.001$ \\
\hline Medical device $^{c}$ & 10.64 & $8.49-13 \cdot 3$ & $<0.001$ & 9.35 & $7.43-11.78$ & $<0.001$ \\
\hline NHSN surgery since admission & 0.98 & $0.83-1.15$ & 0.795 & & & \\
\hline Hospitalisation in intensive care & 4.26 & $3.53-5.15$ & $<0.001$ & 2.50 & $2.10-2.99$ & $<0.001$ \\
\hline Large hospital (>650 beds) & 1.08 & $0.84-1.40$ & 0.542 & & & \\
\hline Tertiary care hospital & 1.00 & $0.78-1.28$ & 0.997 & & & \\
\hline Private-for-profit ownership & 0.92 & $0.70-1.22$ & 0.562 & & & \\
\hline
\end{tabular}

$\mathrm{Cl}$ : confidence interval; NHSN: National Healthcare Safety Network; OR: odds ratio.

age groups: 0-17, 18-40, 41-60, 61-80, >80 years.

${ }^{b}$ Rapidly (within 12 months) or ultimately (within 5 years) fatal disease.

c Peripheral venous catheter, central venous catheter, urinary catheter or endotracheal tube.

Although antimicrobials were prescribed predominantly for therapeutic purposes both in Switzerland and in Europe, there are differences. The proportion of therapeutic indications in Switzerland (63\%) was lower compared with Europe (71\%), while the proportion of prophylaxis, particularly for surgery, was higher $(34 \%$ vs $25 \%$, respectively) [19]. A third of surgical prophylaxis was administered for more than 1 day, particularly in large and tertiary care hospitals, where such use contributed to more than half of surgical prophylaxis. The overall proportion of prolonged surgical prophylaxis, however, was lower than the European average (54\%) and in Germany (54\%), but similar to Poland and Wales $[16,24]$. However, Belgium, the Netherlands, Northern Ireland, Norway and Scotland had lower proportions. By applying best practice recommendations, the contribution of prolonged regimens to surgical prophylaxis could be reduced $[33,34]$.

Amoxicillin/clavulanic acid was the most commonly prescribed antimicrobial not only in Switzerland but also in the EU/EEA, although the contribution of this agent to the total of antimicrobials was much higher in Switzerland (19\% vs $11 \%$ ) [16]. Cefuroxime, ceftriaxone and piperacillin/tazobactam were among the most frequently prescribed antimicrobials in both Switzerland and the EU/EEA, although the proportion of ceftriaxone in Switzerland was higher ( $11 \%$ vs $7 \%$ ). The proportion of broad-spectrum antibiotics in Switzerland was lower than the European average (37\% and $42 \%$, respectively) [16] and in between its neighbouring countries Austria (35\%) and Germany (47\%). There was much variation for drug-combinations; the four most frequent combinations of two agents included metronidazole in combination with a third- or fourth-generation cephalosporin or a fluoroquinolone for the treatment of community-acquired intra-abdominal infections or surgical prophylaxis.

Our survey has limitations. Firstly, prevalence is a point estimate, which limits its generalisability. However, compared with PPS on HAl, a rare outcome, antimicrobial use is a frequent outcome and thus, variation is much lower. Secondly, variation in the application of the definitions on antimicrobial change must be assumed, as challenges with the $\mathrm{CH}$-PPS protocol reported to the coordination centre focused often on interpreting escalation and de-escalation of antimicrobials.

From an organisational point of view, the survey was successful in achieving the desired aims: engaging the majority of Swiss acute care hospitals in a national project, achieving good representativeness of data and 


\section{FIGURE 3}

Prevalence of antimicrobial use in the Swiss and the ECDC point prevalence surveys combined

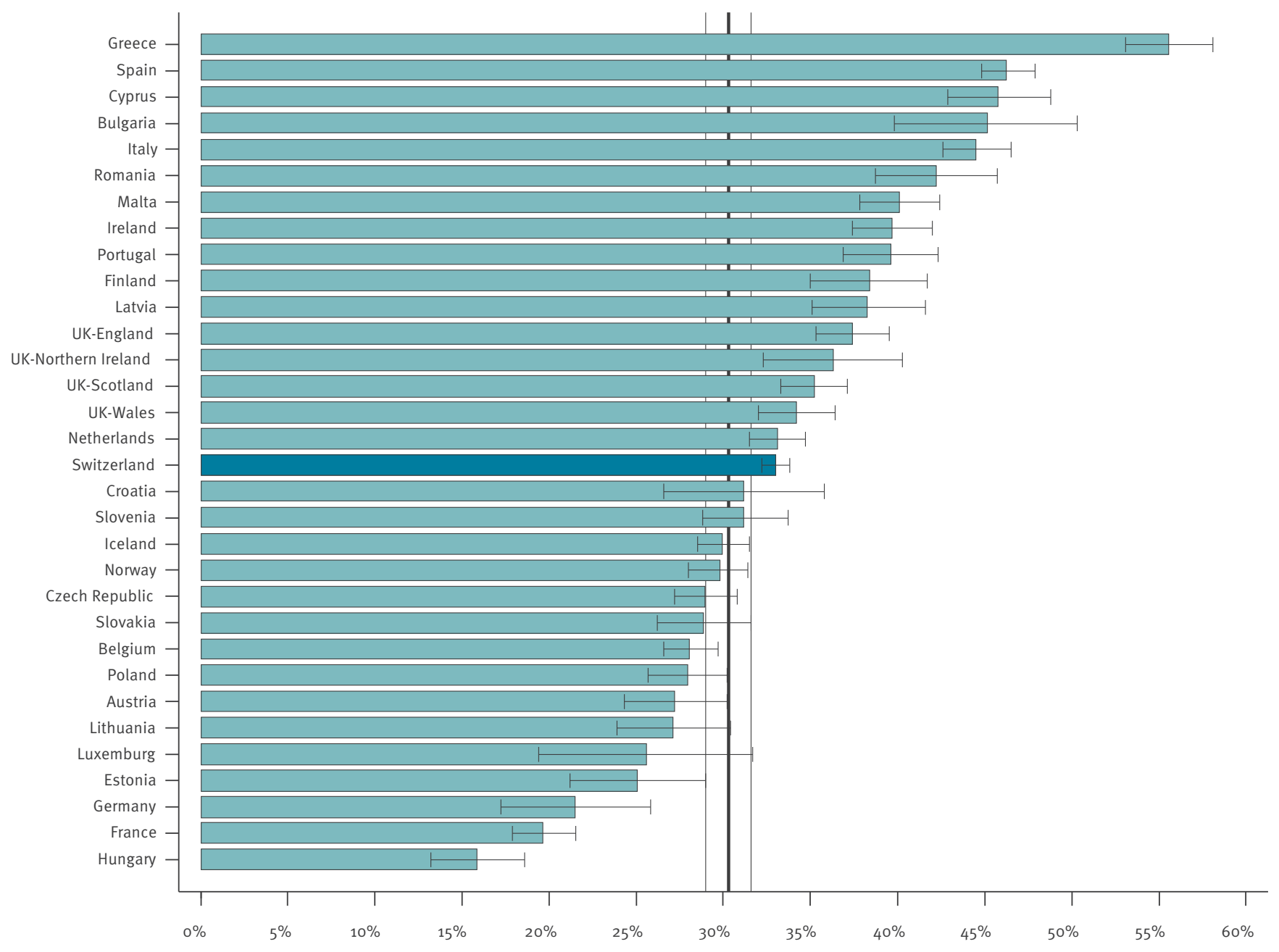

CI: confidence interval; ECDC: European Centre for Disease Prevention and Control; PPS: point prevalence survey.

The prevalence indicates the proportion of patients receiving one or more antimicrobials at time of survey.

Bars and whiskers: prevalences with $95 \% \mathrm{Cl}$ by country. Vertical lines: mean prevalence and $95 \% \mathrm{Cl}$ across the countries participating in the 2016 and 2017 ECDC PPS. Source: [16].

generating data that are comparable with other countries in Europe. Thus, although Switzerland did not formally take part in the second ECDC PPS in 2016 and 2017, applying the ECDC protocol allows benchmarking with EU/EEA countries.

\section{Conclusion}

This was the first national PPS on antimicrobial consumption in Switzerland. Antimicrobial consumption is at European average, the use of broad-spectrum antibiotics in the lower third. Although national surveillance on AMR and antimicrobial consumption has been established, Swiss acute care hospitals should invest in antimicrobial stewardship, particularly in formalising responsibility and post-prescription review. Strategies to reduce antimicrobials, particularly broad-spectrum antibiotics, include the prevention of $\mathrm{HAI}$ and the reduction of prolonged surgical prophylaxis.

\section{Acknowledgements}

We would like to thank all healthcare workers in the participating hospitals, involved in data collection or any other activity in the context of the Swiss point prevalence survey. Also, we would like to thank Viktorija Rion from Swissnoso, and Luis Alberto Pena Diaz from Charité Universitätsmedizin Berlin for their organisational and technical support.

The Swissnoso network consists of the following members: Carlo Balmelli, Marie-Christine Eisenring, Stephan Harbarth, Stefan Kuster, Jonas Marschall, Virginie Masserey Spicher, Didier Pittet, Christian Ruef, Hugo Sax, Matthias Schlegel, Alexander Schweiger, Nicolas Troillet, Andreas Widmer and Giorgio Zanetti. 
Funding: The Swiss prevalence survey was supported by the Federal Office of Public Health as part of the national NOSO strategy [21], and the Antibiotic Resistance Strategy (StAR) [22].

\section{Conflict of interest}

None declared.

\section{Authors' contributions}

WZ contributed to the ECDC PPS protocol as a member of the ECDC PPS team; WZ was the principal investigator of the survey; WZ, AM, and CB planned the survey and wrote the Swiss protocol; WZ, AM, MB CB, CG, AW, NT and DP conducted the survey; WZ did the analysis. WZ wrote the first draft of the manuscript. All authors reviewed and contributed to subsequent drafts and approved the final version for publication.

\section{References}

1. European Centre for Disease Prevention and Control (ECDC). Point prevalence survey of healthcare-associated infections and antimicrobial use in European acute care hospitals 20112012. Stockholm: ECDC; 2013. Available from: https://ecdc. europa.eu/en/publications-data/point-prevalence-surveyhealthcare-associated-infections-and-antimicrobial-use-o

2. Tacconelli E, Sifakis F, Harbarth S, Schrijver R, van Mourik $M$, Voss A, et al. Surveillance for control of antimicrobial resistance. Lancet Infect Dis. 2018;18(3):e99-106. https://doi. org/10.1016/S1473-3099(17)30485-1 PMID: 29102325

3. Willemsen I, Bogaers-Hofman D, Winters M, Kluytmans . Correlation between antibiotic use and resistance in a hospital: temporary and ward-specific observations. Infection. 2009;37(5):432-7. https://doi.org/10.1007/s15010-009-8325-y PMID: 19499184

4. Vernaz N, Huttner B, Muscionico D, Salomon JL, Bonnabry P, López-Lozano JM, et al. Modelling the impact of antibiotic use on antibiotic-resistant Escherichia coli using population-based data from a large hospital and its surrounding community. J Antimicrob Chemother. 2011;66(4):928-35. https://doi. org/10.1093/jac/dkq525 PMID: 21393172

5. Karp J, Edman-Wallér J, Toepfer M, Lundqvist A, Jacobsson G. Clostridioides difficile incidence related to in-hospital cephalosporin use: a tale of two highly comparable hospitals. J Antimicrob Chemother. 2019;74(1):182-9. https://doi. org/10.1093/jac/dky408 PMID: 30358837

6. Wilcox MH, Chalmers JD, Nord CE, Freeman J, Bouza E. Role of cephalosporins in the era of Clostridium difficile infection. J Antimicrob Chemother. 2017;72(1):1-18. https://doi. org/10.1093/jac/dkw385 PMID: 27659735

7. Carignan A, Allard C, Pépin J, Cossette B, Nault V, Valiquette L. Risk of Clostridium difficile infection after perioperative antibacterial prophylaxis before and during an outbreak of infection due to a hypervirulent strain. Clin Infect Dis. 2008;46(12):1838-43. https://doi.org/10.1086/588291 PMID: 18462108

8. Cotten CM, McDonald S, Stoll B, Goldberg RN, Poole K, Benjamin DK Jr, et al. The association of third-generation cephalosporin use and invasive candidiasis in extremely low birth-weight infants. Pediatrics. 2006;118(2):717-22. https:// doi.org/10.1542/peds.2005-2677 PMID: 16882828

9. de Kraker ME, Wolkewitz M, Davey PG, Koller W, Berger J, Nagler J, et al. Burden of antimicrobial resistance in European hospitals: excess mortality and length of hospital stay associated with bloodstream infections due to Escherichia coli resistant to third-generation cephalosporins. I Antimicrob Chemother. 2011;66(2):398-407. https://doi.org/10.1093/jac/ dkq412 PMID: 21106563

10. de Kraker ME, Wolkewitz M, Davey PG, Koller W, Berger J, Nagler J, et al. Clinical impact of antimicrobial resistance in European hospitals: excess mortality and length of hospital stay related to methicillin-resistant Staphylococcus aureus bloodstream infections. Antimicrob Agents Chemother. 2011;55(4):1598-605. https://doi.org/10.1128/AAC.01157-10 PMID: 21220533
11. Stewardson AJ, Allignol A, Beyersmann J, Graves N, Schumacher M, Meyer R, et al. The health and economic burden of bloodstream infections caused by antimicrobialsusceptible and non-susceptible Enterobacteriaceae and Staphylococcus aureus in European hospitals, 2010 and 2011: a multicentre retrospective cohort study. Euro Surveill. 2016;21(33):30319. https://doi.org/10.2807/1560-7917. ES.2016.21.33.30319 PMID: 27562950

12. Baur D, Gladstone BP, Burkert F, Carrara E, Foschi F, Döbele $S$, et al. Effect of antibiotic stewardship on the incidence of infection and colonisation with antibiotic-resistant bacteria and Clostridium difficile infection: a systematic review and meta-analysis. Lancet Infect Dis. 2017;17(9):990-1001. https:// doi.org/10.1016/S1473-3099(17)30325-0 PMID: 28629876

13. Goossens H, Ferech M, Vander Stichele R, Elseviers MESAC Project Group. Outpatient antibiotic use in Europe and association with resistance: a cross-national database study. Lancet. 2005;365(9459):579-87. https://doi.org/10.1016/ S0140-6736(05)70799-6 PMID: 15708101

14. Ansari F, Erntell M, Goossens H, Davey P. The European surveillance of antimicrobial consumption (ESAC) pointprevalence survey of antibacterial use in 20 European hospitals in 2006. Clin Infect Dis. 2009;49(10):1496-504. https://doi.org/10.1086/644617 PMID: 19842976

15. European Surveillance of Antimicrobial Consumption Network (ESAC-Net). Summary of the latest data on antibiotic consumption in the European Union. ESAC-Net surveillance data. Stockholm: European Centre for Disease Prevention and Control; 2017. Available from: https://ecdc.europa.eu/ sites/portal/files/documents/Final_2017_EAAD_ESAC-Net Summary-edited\%20-\%20FINALwith\%20erratum.pdf

16. Plachouras D, Kärki T, Hansen S, Hopkins S, Lyytikäinen O, Moro ML, et al. Antimicrobial use in European acute care hospitals: results from the second point prevalence survey (PPS) of healthcare-associated infections and antimicrobial use, 2016 to 2017. Euro Surveill. 2018;23(46). https://doi. org/10.2807/1560-7917.ES.23.46.1800393 PMID: 30458917

17. Zingg W, Hopkins S, Gayet-Ageron A, Holmes A, Sharland $M$, Suetens $C$, et al. Health-care-associated infections in neonates, children, and adolescents: an analysis of paediatric data from the European Centre for Disease Prevention and Control point-prevalence survey. Lancet Infect Dis. 2017;17(4):381-9. https://doi.org/10.1016/S1473 3099(16)30517-5 PMID: 28089444

18. European Centre for Disease Prevention and Control (ECDC). Point prevalence survey of healthcare-associated infections and antimicrobial use in European acute care hospitals: protocol version 5.3. Stockholm: ECDC; 2016. Available from: https://ecdc.europa.eu/en/publications-data/pointprevalence-survey-healthcare-associated-infections-andantimicrobial-use-3

19. Suetens C, Latour K, Kärki T, Ricchizzi E, Kinross P, Moro $M L$, et al. The Healthcare-Associated Infections Prevalence Study Group. Prevalence of healthcare-associated infections, estimated incidence and composite antimicrobial resistance index in acute care hospitals and long-term care facilities: results from two European point prevalence surveys, 2016 to 2017. Euro Surveill. 2018;23(46). https://doi. org/10.2807/1560-7917.ES.2018.23.46.1800516 PMID: 30458912

20. Swiss Federal Office of Public Health (FOPH). Gesundheit 2020 - Health 2020 - a comprehensive health care strategy. Liebefeld: FOPH; 2013. Available from: https://www.bag.admin. ch/bag/en/home/strategie-und-politik/gesundheit-2020/eineumfassende-strategie-fuer-das-gesundheitswesen.html

21. Swiss Federal Office of Public Health (FOPH). National strategy for the monitoring, prevention and control of healthcareassociated infections (NOSO Strategy). Liebefeld: FOPH 2016. Available from: https://www.bag.admin.ch/bag/en/home/dasbag/publikationen/broschueren/publikationen-uebertragbarekrankheiten/publikation-nationale-strategie-noso.html

22. Swiss Federal Office of Public Health (FOPH). Strategy on antibiotic resistance (StAR). Liebefeld: FOPH; 2015. Available from: https://www.bag.admin.ch/bag/en/home/das-bag/ publikationen/broschueren/publikationen-uebertragbarekrankheiten/strategie-anbitiotikaresistenzen-schweiz.html

23. Metsini A, Vazquez M, Sommerstein R, Marschall J, Voide C, Troillet N, et al. Point prevalence of healthcare-associated infections and antibiotic use in three large Swiss acute-care hospitals. Swiss Med Wkly. 2018;148:w14617. PMID: 29698542

24. Behnke M, Aghdassi SJ, Hansen S, Diaz LAP, Gastmeier $P$, Piening $B$. The prevalence of nosocomial infection and antibiotic use in German hospitals. Dtsch Arztebl Int. 2017;114(50):851-7. https://doi.org/10.3238/arztebl.2017.0851 PMID: 29271343

25. Zarb P, Ansari F, Muller A, Vankerckhoven V, Davey PG, Goossens H. Drug utilization 75\% (DU75\%) in 17 European 
hospitals (2000-2005): results from the ESAC-2 Hospital Care Sub Project. Curr Clin Pharmacol. 2011;6(1):62-70. https://doi. org/10.2174/157488411794941322 PMID: 21235461

26. World Health Organization (WHO). ATC/DDD Index 2019 Geneva: WHO. [Accessed: 1 Apr 2019]. Available from: https:// www.whocc.no/atc_ddd_index/

27. European Centre for Disease Prevention and Control (ECDC), European Food Safety Agency (EFSA), European Medicines Agency (EMA). ECDC, EFSA and EMA Joint Scientific Opinion on a list of outcome indicators as regards surveillance of antimicrobial resistance and antimicrobial consumption in humans and food-producing animals. EFSA J. 2017;15(10):5017.

28. McCabe WR, Jackson GG. Gram-negative bacteremia. I. Etiology and ecology. Arch Intern Med. 1962;110(6):847-53. https://doi. org/10.1001/archinte.1962.03620240029006

29. National Healthcare Safety Network (NHSN). Surgical site infection (SSI) event. Atlanta: Centers for Disease Control and Prevention. [Accessed 12 Feb 2019]. Available from: https:// www.cdc.gov/nhsn/PDFs/pscManual/9pscSSIcurrent.pdf

30. Zingg W, Metsini A, Balmelli C, Neofytos D, Behnke M, Gardio $C$, et al. Findings of the first national point prevalence survey on healthcare-associated infections in acute care hospitals in Switzerland. Euro Surveill.2019;24(33):1800603.

31. Natsch S, Hekster YA, de Jong R, Heerdink ER, Herings RM, van der Meer JW. Application of the ATC/DDD methodology to monitor antibiotic drug use. Eur J Clin Microbiol Infect Dis. 1998;17(1):20-4. https://doi.org/10.1007/BF01584358 PMID: 9512177

32. Anresis. Hospital care. Bern: Institut für Infektionskrankheiten. [Accessed: 1 Apr 2019]. Available from: http://www.anresis.ch/ index.php/hospital-care.html

33. Allegranzi B, Bischoff P, de Jonge S, Kubilay NZ, Zayed B, Gomes SM, et al. New WHO recommendations on preoperative measures for surgical site infection prevention: an evidencebased global perspective. Lancet Infect Dis. 2016;16(12):e27687. https://doi.org/10.1016/S1473-3099(16)30398-X PMID: 27816413

34. Allegranzi B, Zayed B, Bischoff P, Kubilay NZ, de Jonge S, de Vries $\mathrm{F}$, et al. New WHO recommendations on intraoperative and postoperative measures for surgical site infection prevention: an evidence-based global perspective. Lancet Infect Dis. 2016;16(12):e288-303. https://doi.org/10.1016/ S1473-3099(16)30402-9 PMID: 27816414

\section{License, supplementary material and copyright}

This is an open-access article distributed under the terms of the Creative Commons Attribution (CC BY 4.0) Licence. You may share and adapt the material, but must give appropriate credit to the source, provide a link to the licence and indicate if changes were made.

Any supplementary material referenced in the article can be found in the online version.

This article is copyright of the authors or their affiliated institutions, 2019. 